\title{
Research Progress on Wound Healing of Perianal Necrotizing Fasciitis in Traditional Chinese Medicine
}

\author{
Shufa Tan \\ Affiliated Hospital of Shaanxi University of Chinese Medicine Xianyang 712000, Shaanxi, China
}

\begin{abstract}
Perianal necrotizing fasciitis is a potentially life-threatening, explosive infectious disease that is difficult in early diagnosis and develops rapidly. It is often accompanied by systemic toxic shock and a large number of toxins even after the operation. After thorough wound surgery, an extensive open wound will form, deep and heal slowly. How to remove the residual toxins in the body after surgery, reduce the patient wound pain, shorten the healing time and improve the healing rate is the problem that clinicians need to solve at present. Traditional Chinese medicine in the treatment of wound healing has a unique theoretical basis and advantages, this paper systematically combed the recent literature on the PNF wound healing, respectively from traditional Chinese medicine, traditional Chinese medicine sitting bath, fumigation, Chinese medicine, Chinese medicine characteristic treatment to discuss and evaluation, put forward the problems existing in traditional Chinese medicine treatment, and look forward to promote PNF wound healing treatment.
\end{abstract}

Keywords: Perianal necrotic fasciitis, TCM treatment, Wound healing, research progress, Review.

\section{Introduction}

Perianal necrotizing Fasciitis (PNF) is an acute infectious disease caused by mixed infection of aerobic and anaerobic bacteria[1], Studies have shown that[2] the most common pathogen of PNF is Group A streptococcus. After the onset of the scrotum, perineal trigone, soft tissue skin structure around the anus and deep fascia. Under the common influence of bacterial invasion and low immune function of the body, the subcutaneous tissue and fascia of the lesion site will be rapidly and extensively necrotic, generally not affecting the muscle of the lesion site, and there may be a sense of holding snow locally, and the sound of twirling can be heard. Rapid onset of the disease, early may only appear to crissum local erythema mainly associated with fever symptoms mild inflammation, easily diagnosed as delay the best operation time of anus week abscess, progress rapidly, treatment is not timely development perineum, abdominal infection easily[3], and severe complications, often visible blood coagulation dysfunction, sepsis, or sepsis, Cause systemic toxic shock, multiple organ failure, life-threatening. Although the incidence of the disease is low in epidemiology, the mortality rate is $20 \%-40 \%$ higher[4], and it is more common in males[5]. Treatment principle is given priority to with early surgical removal of necrotic tissue thoroughly, postoperative mainly anti-infective support therapy, but due to large surgical trauma, crissum wound deeper and irregular, and crissum anatomical location vulnerable to stimulation of excrement and urine, which appear in postoperative patients with different degree of wound bleeding, edema, pain, poor prognosis[6]. Postoperative treatment of traditional antibiotics combined with anti-infection treatment and wound drainage, but it often cannot reduce postoperative local pain patients, and postoperative wound healing time longer, easy to wound large scar tissue formation, some scholars study[7] the long-term use of antibiotics may cause systemic adverse reactions (lead to intestinal disorders, the body resistance), and a poor prognosis. Traditional Chinese medicine has a unique advantage in promoting wound healing that is more effective than western medicine, especially in eliminating decay and discharging pus, detoxifying muscles, detumescence and analgesia, etc., which can enhance the immune capacity of the body through internal and external treatment of drugs, so as to promote wound healing.

\section{Understanding of Perianal Necrotizing Fasciitis and Wound Healing in Traditional Chinese Medicine}

\subsection{Understanding of Perianal Necrotizing Fasciitis in Chinese Medicine}

Traditional Chinese medicine believes that the supurulent tissue between tendon and bone is gangrenous syndrome[8], so perianal necrotizing fasciitis belongs to "crotch-wear gangrenous", "marabou gangrenous", "rotten malignant", "dirty poison", "ulcer disease" and other categories[9], some scholars according to the characteristics of the disease to "anal poison" named this disease. This disease is mainly caused by patients' weak body, insufficient positive qi, infection of external poison, fiery fire poison, being overcome by evil, inability to go out with evil, instead falling into the internal organs, and the disease is dangerous, the onset of rapid, easy to occur yellow heavy syndrome, and then endanger life safety[10].

\subsection{Understanding of Wound healing in TCM}

The exposed wound after ulceration is similar to the infectious wound in modern medicine, and the postoperative wound recovery process of perianal necrotizing fasciitis is equivalent to the post-ulceration recovery period in TCM[11]. According to the theory of traditional Chinese medicine, the spleen plays a key role in transporting the essence of water, grain and body fluid, generating qi and blood to nourish and nourish the muscles. If qi, blood, jin and essence are abundant, the muscles will be plump and powerful, and the skin will be moist. Lack of temper is low immunity of the body, the ability to remove toxins in the body is reduced, easy to cause wound infection. Therefore, qi and blood are needed to promote the 
healing of wounds. It can be seen that the recovery of wounds in the late stage of ulceration is closely related to the fluctuation and decline of qi and blood, and the operation of qi and blood.

\section{TCM Treatment}

Although PNF wounds are localized in perianal skin, the occurrence, development and change of wounds are closely related to the dysfunction of viscera[12]. The operation hurts the body's meridians and veins, and causes the loss of lung, spleen and kidney[13], the deficiency of qi and blood, the damage of healthy qi, and the inability to drum evil to go out. Damp-heat residual poison, poison, heat, blood stasis, deficiency and other evil gathered in the wound, blocked blood vessels, pain, so patients are prone to anal bleeding, edema, pain, slow growth of granulation and wound delayed healing or non-healing. The general principle of treating sore in TRADITIONAL Chinese medicine is "eliminating, supporting and supplementing", treating according to syndrome differentiation, treating both inside and outside, and treating different rules and prescriptions in different stages of the disease.

\subsection{Internal TCM Treatment}

\subsection{1"Elimination method"}

Use prescription to dissipate dampness and heat residual poison and avoid the accumulation of poison and evil. Congying et al.[1] believe that the swelling and toxin of ulcers are mostly formed by accumulation of heat and toxin, and toxic evil is held in skin cou rationale, which leads to slow recovery of the wound. Treatment should be carried out to clear heat and detoxification, and carry out qi to disperse the knot and destroy the foundation. After taking Xianfang Huoxinyin, the wound edema and pain can be effectively alleviated. The wound healing rate of the treatment group is significantly faster than that of the control group. The treatment can inhibit the infection of bacteria on the wound, significantly promote the secretion of growth factors and accelerate the generation of granulation tissue[2]. Xianfang Huoxingyin can clear heat and detoxify, promote qi, promote blood circulation and relieve pain, detumescence and dissipate swelling, and has good antibacterial and anti-inflammatory effects. Honeysuckle in the prescription is known as the "holy medicine for sore" in classical Chinese medicine, because it tastes sweet and cold, and is the best heat-clearing and detoxifying treatment for sore[14]. Feng Xiuli et al.[15] found in the study that Honeysuckle and its compound Xianfang Huizhao Decoction had an obvious inhibitory effect on bacteria, and both of them could eliminate xylene induced auricle swelling in mice to a certain extent, indicating that inflammation could be eliminated. Therefore, the use of honeysuckle single drug or combination can effectively inhibit the generation of inflammatory mediators and improve the immune capacity of the body, which plays a great role in promoting wound healing after PNF. Although patients with perianal necrotizing fasciitis underwent surgical debridement, postoperative inflammation was severe, and there were still dampness, heat and toxic evil in the body, often presenting symptoms such as dry mouth, chest tightness and upset. The effect of western medicine fluid rehydration and anti-inflammatory treatment was not obvious. Tang Xiaoru[16] observed 81 cases of patients with primary or secondary fever treated by adding and reducing Bamboo Yegypsum decoction. It was found that Chinese medicines (bamboo leaves, gypsum, Ophiopogon, etc.) can clear qi, heat, qi and stomach, treat gangx and PNF, with a total effective rate of $93.84 \%$. It can be seen that bamboo Yegypsum decoction has excellent efficacy in treating postoperative complications of PNF.

\subsection{2 "Tuofa"}

Use drugs that are permeable with pyotosis to make the poison go out and avoid the spread and even internalization of the poison.Ma Le et al.[17]believed that in order to make the wound heal faster and relieve edema, pain and inflammation, it should be used to clear heat, detoxify, detumescence and discharge pus as the treatment method. He divided 108 patients with skin ulcer into two groups, 54 patients in the control group were treated with Xiangpi Shengji ointment, and 54 patients in the research group were treated with oral Tori disinfection liquid on the basis of shengji ointment. Results: The total effective rate in the study group was $94.44 \%$ significantly higher than $75.93 \%$ in the control group, indicating that oral Tori can effectively reduce the inflammatory factors in serum, improve the purification rate of rotting meat, accelerate the formation of new granulation tissue and promote wound healing. Liang Yongsheng et al.[18] observed the wound healing of perianal necrotizing fasciitis by using Todu Yiqi Yangyin Decoction in random groups, and found that this recipe could reduce the swelling area of the wound, increase the blood hemoglobin, accelerate the growth rate of epidermal granulation tissue, reduce the hospitalization time and reduce the hospitalization cost of patients. Gu Feng[19] observed the degree of postoperative pain of perianal necrotic fasciitis improved by transpyototoxin Decoction (Astragalus, Forsythia, Fritillaria, Trichosanthes, Chuanqiong, pangolin, etc.). 28 patients in the control group were treated with conventional surgery, and 29 patients in the treatment group were treated with transpyototoxin decoction orally on the basis of conventional treatment[20]. Clinical observation showed that perianal pain in the treatment group $(6.83 \pm 1.42$ before treatment; The degree of $1.51 \pm 0.39$ after treatment was significantly lower than that of the control group ( $6.76 \pm 1.29$ before treatment; $2.34 \pm 0.66$ after treatment), the difference was statistically significant. Forsythia in qingrejiedu, angelica, nourishing blood and blood compatibility of rhizoma ligustici wallichii, the medicine compatibility can effectively reduce the swelling pain, anti-inflammatory analgesic, and promote the growth of granulation tissue and rotten meat pieces, can be wound may produce range of lesions, improve curative effect and reduce postoperative complications and accelerate the wound healing, reduce the physiological burden of patients. Through septic sores poison drinking party Chinese medicine astragalus to home yiqi poison the effective medicine, in the treatment of ulcers long collapse of deficiency of qi and blood, have a good role in painless, Xie Jing[21] in astragalus membranaceus on sepsis in the clinical research progress of astragalus membranaceus has antiviral and antibacterial effect, can promote angiogenesis in the treatment of sepsis make smooth the blood circulation, regulate inflammation factor levels, improve the body's immune function, Thus effectively 
prevent sepsis and inhibit inflammatory response.

\subsubsection{Tonic method}

The use of drugs supplementing qi and blood can help the body to restore the vital qi, which can drum out evil spirits, accelerate the decay of flesh and promote the healing of wounds. Perianal necrotizing fasciitis is bound to consume a large amount of qi, blood and body fluid due to its serious condition, long course and surgical detrauma. It is of great benefit to use qi and blood tonic drugs after the operation for wound healing of patients. Cao rangfu[22] think gold blade trauma can damage human body jet, temper is damaged, the source is insufficient, the deficiency of the transit to cause constipation hard, can line taken blood, blood gas, unable to promote the circulation of the blood which vital qi operation impeded, cause anus week local qi and blood stasis, and the operation process a large amount of blood loss, blood deficiency, no glory, pain. He applied Buzhong Yiqi Decoction (Astragalus membranaceus, Cohoma, Bupleurum, Angelica, Atractylodes atractylodes, Dangshen, etc.) to treat wound healing after anorectal diseases. Results: The effective rate of traditional Chinese medicine group was $97.87 \%$, and the effective rate of western medicine group was $89.36 \%$. The traditional Chinese medicine group was better than western medicine group in alleviating postoperative edema and pain, reducing the amount of blood loss, and keeping the stool unblocked. Li Liangzeng[23] from the perspective of Traditional Chinese medicine, believed that poor wound healing was mostly caused by deficiency of qi and blood and obstruction of channels and collaterals. He applied Shishidadu Decoction to patients after anal fistula surgery and found that: Compared with the conventional treatment group, the incidence of postoperative complications was significantly reduced and the wound healing rate was accelerated after taking Shiquandabu Decoction, which proved that Shiquandabu Decoction could regulate qi and blood, remove blood stasis and build muscle, improve the local immune function of patients and promote wound healing.

\subsection{External Treatment of Chinese medicine}

Modern medical study found that the healing of wound and nascent capillaries and fibroblasts granulation tissue and epithelial tissue regeneration[24], have close relations, according to the skin has the characteristics of absorption and penetration, evacuation, when wound surrounding skin exposure to drugs, drug ion can be through the skin penetration into the blood circulation, This local administration allows the drug to be delivered directly to the target site, while avoiding gastrointestinal tract irritation. Drug ions penetrate into local tissues through skin capillaries, which is conducive to fibrin dissolution and the shedding of necrotic tissues. Chinese medicine believes that local skin can drege channels and collaterals, harmonize qi and blood, support poison to go out, and nourish and dispel evil, through absorption and penetration of drug ions. Thus, it can effectively inhibit the bacterial infection on the wound surface, reduce the release of inflammatory factors, relieve pain, accelerate the regeneration of epithelial tissue and granulation tissue, and promote the wound healing.

\subsubsection{Traditional Chinese medicine sitting bath}

The scholar thinks, in the moist environment swimming and migration of cells to a dynamic, moist wound environment can effectively keep the shallow growth factor, stimulates the production of blood capillary, accelerate the growth of fiber cells, moist environment increase the skin moisture content, corneous layer is easier to make porous state drug ion penetrating into the systemic circulation[25]so as to promote the healing of wounds, In addition, the enzyme activity can be maintained in the moist environment, so that the tissue proteolytic enzyme in the wound exudate can effectively dissolve the necrotic tissue and fibrin, reduce the chance of bacterial invasion and reduce the risk of infection. Traditional dry dressing change medicine to cause tissue adhesion, the treatment can often increase the patients' pain, and tend to form a large area of scar tissue after wound healing, and wound environment can reduce the dry scab formation so as to avoid the disturbance of newborn granulation tissue scab caused by mechanical damage[26], patients can effectively reduce pain, to reduce the risk of the wound. Chinese medicine sitz bath can not only create good moist environment for wound, also can make the drug direct lesions, warm sitz bath can increase local skin temperature, make the hair follicles, sweat glands and sebaceous glands opening, increase the ion penetration and absorption of drugs, promote the blood circulation, accelerate the elimination of the metabolites, inflammation medium, and alleviate the pain and accelerate wound healing. Li Yubo[27] that PNF to go saprophytic muscle after surgery, invigorate the circulation of detoxification treatment, he used the medicine juice temperature of $40^{\circ} \mathrm{C}$ about the hard work of soup (sophora, top ten credit, alum, two needles, etc.) line sitz bath to adjuvant treatment crissum necrotizing fasciitis postoperative wound healing, found sitz bath group total effective rate was $100 \%$ higher than that of conventional group total effective rate $69.2 \%$, It can promote local blood circulation, inhibit bacterial proliferation, accelerate the shedding of rotting flesh and promote the release of growth factors to form normal tissue skin, through modern pharmacological studies[28], it has been found that alkaloids, the main chemical components in its stems and leaves, have good antibacterial and anti-inflammatory effects. Qiao Fengni[29] applied the heat clearing, detoxification, swelling and pus clearing formula to sit bath, and found that the traditional Chinese medicine sitting bath can effectively relieve patients' pain, promote blood circulation, improve congestion and edema, and accelerate wound healing.Hu Zhiliang[30] found in a randomized controlled study that local sit-bath with Detumescence and Zhitong prescription after PNF debridement could significantly promote wound healing.

\subsubsection{Fumigation and washing of Traditional Chinese medicine}

Zhang Xiuling[31] compared the recurrence rate after PNF between conventional treatment and traditional Chinese medicine fumigation. She randomly divided 100 patients with necrotizing fasciitis around anus into two groups with 50 patients in each group, who received conventional treatment and fumigation with Jinghuang Decoction respectively. The results showed that the length of hospital stay in the fumigation group $(35.7 \pm 5.0) \mathrm{d}$ was much lower than that in the conventional treatment group $(52.1 \pm 7.2) \mathrm{d}$. The recurrence 
rate of patients using Chinese herbal fumigation was 0.Wang Lehui[32] in a randomized controlled studies compared the hydrogen peroxide rinse and the analgesic effect of compound cortex phellodendri fluid flush after surgery, compared with pure wash the wound with hydrogen peroxide solution, hydrogen peroxide with compound cortex phellodendri liquid rinse can effectively reduce postoperative pain in patients with alternately, improve edema, reduce the heating rate, reduce postoperative infection patients, control time and average period of treatment. It is proved that the use of Traditional Chinese medicine fumigation can effectively inhibit bacterial infection, improve the blood circulation around the anal end, and play an important role in shortening the hospitalization time and reducing the recurrence rate after discharge, which undoubtedly reduces the economic burden and physical pain of patients.

\subsubsection{External application of TCM}

Traditional Chinese medicine believes that the process of wound healing should be "simmering pus and growing flesh" as the treatment method. In the process of wound recovery, if the poison can not be dissipated, it will gather on the wound and form rotting flesh. The ancient doctor Xue Ji pointed out that only when rotting flesh goes, new flesh will grow. When Chinese traditional medicine ointment is used locally, it can accelerate the exudation of pus on the wound surface under the pharmacological action, thus carrying pathogens out, and play the role of discharging pus, removing rot and building muscle. Modern medical research has found that Chinese traditional medicine ointment has a strong bacteriostatic and bactericidal effect on a variety of bacteria including Escherichia coli, Staphylococcus aureus and Proteus. This mechanism is similar to the wound healing mechanism studied in modern medicine: (1) removing pathogenic microorganisms from the body and regulating the mechanism of inflammatory response; (2) improving the blood circulation around the wound tissue, maintaining the hypoxic microcirculation environment on the wound and creating the growth environment of granulation tissue[33]. Traditional Chinese medicine believes that the process of wound healing should be "simmering pus and growing flesh" as the treatment method. In the process of wound recovery, if the poison can not be dissipated, it will gather on the wound and form rotting flesh. The ancient doctor Xue Ji pointed out that only when rotting flesh goes, new flesh will grow. When Chinese traditional medicine ointment is used locally, it can accelerate the exudation of pus on the wound surface under the pharmacological action, thus carrying pathogens out, and play the role of discharging pus, removing rot and building muscle. Modern medical research has found that Chinese traditional medicine ointment has a strong bacteriostatic and bactericidal effect on a variety of bacteria including Escherichia coli, Staphylococcus aureus and Proteus. This mechanism is similar to the wound healing mechanism studied in modern medicine: (1) removing pathogenic microorganisms from the body and regulating the mechanism of inflammatory response; (2) improving the blood circulation around the wound tissue, maintaining the hypoxic microcirculation environment on the wound and creating the growth environment of granulation tissue[34] observed the clinical efficacy of ziyu Ointment (Crassifolia chinensis, Ulmus officinalis, Borneol, Canicera sinensis, Angelica angelica and Angelica dahurica) in promoting wound healing after PNF, and found that after 14 days, the increase of serum VEGF level in the treatment group was higher than that in the control group (4.32 $\pm 0.46: 3.42 \pm 0.55)$, il-8 level decreased more than the control group $(0.32 \pm 0.15 / 0.45 \pm 0.11)$. It proved that the application of Ziyu Ointment after surgery can create a wet environment, promote the effective absorption of traditional Chinese medicine ions in the wound surface, thus increasing vascular permeability; meanwhile, the increase of VEGF can accelerate the increment of cells, leading to the rapid growth of granulation tissue; the decrease of IL- 8 can reduce the aggregation of inflammatory cells, thus alleviating the pain sensation of patients. In summary, the treatment of "simmering pus and long flesh" can improve local microcirculation, reduce postoperative complications and improve wound healing rate. NieMin[35]think traditional surgical dressing PNF postoperative wound healing as well, she's in a randomized controlled study discusses the moist burn cream (rhizoma coptidis, cortex phellodendri, radix scutellariae, etc.) for PNF degree of postoperative pain relief and wound healing rate, it is found that Chinese medicine wound healing rate of the treatment group was significantly higher than that of control group, anus week local pain degree is lighter than the control group obviously. Some scholars have found that[36]a large amount of baicalein contained in MEBO can inhibit bacterial reproduction, reduce the release of inflammatory factors and improve local microcirculation. In conclusion, the external application of Traditional Chinese medicine ointment can significantly promote wound healing, relieve the pain of patients, and the therapeutic effect is significantly better than the traditional surgical dressing change.

\subsection{TCM Characteristic Treatment}

\subsubsection{Moxibustion}

Moxibustion refers to moxa, lit by a slow continuous heat directly or indirectly from the burning of fume burning local surface of traditional Chinese medicine treatment, traditional Chinese medicine think moxibustion WenTong meridian, YangJu trapped, fights the action such as qi huoxue, detumescence, clinical can be divided into direct moxibustion, indirect moxibustion and moxa roll moxibustion, moxibustion box of several ways. Modern medicine thinks: local warm heat stimulation can cause cerebral cortical inhibitory material to spread, reduce the excitability of nervous system, achieve calm thereby, the effect of acetanilide. Some scholars have found[37], that moxa leaves in moxibustion have an inhibitory effect on a variety of bacteria, which can significantly increase the body's $\mathrm{T}$ cell population so as to improve the body's immune capacity and effectively fight infection. Kan $\mathrm{Yu}$ [38] found in a study on the healing effect of moxibustion on skin trauma that moxibustion can promote the infiltration of inflammatory cells and the increment of fibroblasts in the inflammatory reaction stage of wound, regulate local blood circulation and promote wound healing. Wang XY[39] by observing the acupoints moxibustion combined wound moxibustion for anal fistula postoperative wound healing, the influence of the results found that acupuncture moxibustion combined wound moxibustion postoperative wound healing rate is significantly higher than the control group, can effectively promote wound granulation 
tissue growth, improve the healing quality, adjust the content of collagen to avoid excessive hyperplasia and reduce scar formation after healing.

\subsubsection{Acupuncture}

Traditional medicine thought that acupuncture acupoints have purging fire detoxification, anti-inflammatory analgesic, aperient effect such as urine, PNF often because the wound pain after operation in patients with defecation difficulties, stool cause mechanical damage of wound, in the process of wound healing and easily lead to wound infection, thus maintain defecate unobstructed after surgery is important for prognosis.Yang Zhen[40] observed the defecation patency of patients with hemorrhoid acupoints (Hegu, Zusanli, Shang Juxu, Daheng, etc.) after acupuncture with mixed hemorrhoids. The results showed the time of first defecation after acupuncture $(35.64 \pm 13.74) \mathrm{h}$ was significantly faster than that of the control group $(48.18 \pm 14.93) h$, and the defecation in the acupuncture group was more patency than that in the control group. Compared with oral drug therapy, it can effectively reduce postoperative complications and accelerate wound healing. Acupuncture acupoints also has the function of regulating blood vessels, increase blood supply, reducing the role of inflammation, wound scholars Gai Juanjuan[41] through the use of acupuncture wang singular point (the door hole, the hole, it is point) combined traditional Chinese medicine hip bath of mixed hemorrhoid postoperative wound healing, observed: acupuncture group wound pain was significantly lower than the control group (1.12+/-0.04:3.04 \pm 0.72$)$, serum bFGF (cell growth factor) and VEGF (vascular endothelial growth factor) were significantly increased in acupuncture group. Some studies have shown that[42] VEGF can promote angiogenesis and improve vascular permeability. After angiogenesis, it can provide abundant blood supply for wound, so as to restore ischemic tissue and promote wound healing. Liu Xiaotong[43] explored the effects of VEGF and bFGF on wound healing in rats and found that bFGF could accelerate the formation of new blood vessels, promote the growth of fibroblasts, and promote collagen synthesis by acting on smooth muscle cells and endothelial cells to improve wound healing ability. The increase of VEGF can establish good blood circulation in local perianal wounds and provide material basis for the formation of granulation tissue. BFGF is related to the proliferation of scar tissue and complete regeneration of epidermis, so the increase of bFGF is helpful to reduce the wound area. In conclusion, VEGF and bFGF synergistically promote neovascularization and thus promote the repair and regeneration of wound tissues. Zhang Jin[44] observed in a randomized controlled experiment that acupuncture stimulation can promote the release of neuropeptide SP from peripheral nerves, which is an important medium for wound healing. The increase of neuropeptide SP content can promote the growth of fibroblasts, promote the proliferation of vascular endothelial cells and finally complete wound healing.

\section{Problems and Prospects}

Perianal necrotizing fasciitis belongs to the category of "sore" in Traditional Chinese medicine. The disease has a sudden onset, rapid progression and high mortality. Therefore, surgical treatment should be carried out quickly after early diagnosis. TCM holds that, in addition to the PNF postoperative lesions, but also create new wound, postoperative vital qi deficiency, and hot and humid FKK, not poison, heat, blood stasis, virtual gathered in the wound, blood is blocked, general clauses pain, so patients prone to anal bleeding, edema, pain, slow growth of the granulation and delayed union or healing of the wound. Through the study of relevant literature at home and abroad in recent years, the use of traditional Chinese medicine in postoperative treatment "internal and external treatment (oral clearing heat and detoxification, qi sanjie, tonifying qi and blood, invigorating qi and spleen; the treatment methods such as sit-bath, fumigation, external application, acupuncture and moxibustion etc.)" can effectively remove the residual toxins in the body, relieve the pain of the wound, shorten the healing time and improve the healing rate. However, there are still shortcomings in the current TCM treatment methods: (1) TCM doctors have strong subjectivity in dialectical classification of diseases, and different doctors may identify different syndrome types, lacking unified standards. (2) There are many kinds of drugs and complex ingredients in traditional Chinese medicine, and the specific ingredients that play a role in promoting wound healing cannot be fully understood. (3) Traditional Chinese medicine sitting bath and fumigation are inconvenient to carry and need to be treated in a specific treatment room. But in general, the advantages of TCM therapy, such as simple operation, low treatment cost and fewer side effects on patients, are worthy of clinical promotion. In the future we should be based on the existing research results, in the unity of traditional Chinese medicine diagnosis standard, clear mechanisms of traditional Chinese medicine to promote wound healing, deeply researching the basic experiment of traditional Chinese medicine (TCM), the effective extraction of TCM effective constituents, etc to do further research, to promote the traditional Chinese medicine in crissum necrotizing fasciitis of participation in the process of treatment, better play to the advantages of traditional Chinese medicine and curative effect.

\section{References}

[1] Guo Congying, Li Shuaijun. Effect of Xianfang Huoxingyin oral administration combined with hydrogen peroxide irrigation on wound healing of perianal necrotizing fasciitis[J]. Asia-pacific Traditional Medicine, 2020, 16(12): 168-171.

[2] Walkermj, Barnetttc, Mcarthurjd, et al. Disease manifestions and pathogenic mechanisms of groupa streptococcus[J]. Clinical Microbiology Reviews, 2014, 27(2): 264

[3] Liu Ruirui, Cao Yongqing, Yao Yibo. Chinese Journal of Integrated Traditional and Western Medicine Surgery, 2020, 26(02): 382-385.

[4] Lee MH, Votto SS, Read-Fuller AM, et al. Necrotizing fasciitis of the scalp stemming from odontogenic infection[J]. Proc (Bayl Univ Med Cent). 2019,33(1):110-112

[5] Ersay A, Yilmaz G, Akgun Y, et al. Factors affecting mortality of Fournier's gangrene: review of 70 patients[J]. ANZ Journal of Surgery, 2007, 77(1-2): 43-48. 
[6] Li chunyu. Clinical manifestations and management principles of perianal necrotizing fasciitis[J]. Chinese Journal of Colorectal Diseases Electronic Journal, 2013, 2(04): 151-153.

[7] Zhang Xinlong, Shi Jian, Wu Yue, et al. Journal of Anhui University of Traditional Chinese Medicine, 2018, 37 (5): 11-14

[8] Zhang Zhi-yun, Wang Xiao-lan, Yang Wen-zhi, ZHU Yun-ying, WANG Jian-da. Treatment of large area necrotizing fasciitis secondary to perianal abscess[J]. Yunnan Journal of Traditional Chinese Medicine and Chinese Materia Medica, 2018, 39(08): 40-41.

[9] Zhang Yan, Ma Fuming, Li Feng. Clinical experience of perianal necrotizing fasciitis[J]. Inner Mongolia Traditional Chinese Medicine, 2019, 38(07): 84-86.

[10] Fu Yang, Han Tao. Effect of external Chonghe Ointment combined with Compound Phellodendron liquid wound lavage on wound healing after perianal necrotizing fasciitis[J]. Journal of Clinical Military Medicine, 2021, 49(01): $25-27+30$.

[11] Li Daigan. Clinical efficacy evaluation of jiawei Tori Disinfection Powder for postoperative low perianal abscess[D]. Chengdu University of Chinese Medicine, 2020.

[12] Zheng Chunju, Wang Yehuang. Research progress of traditional Chinese medicine on wound healing after anal fistula[J]. World Journal of Integrated Traditional and Western Medicine, 2014, 9(02): 207-209.

[13] Xu Xiang, Wang Chen, Lu Jin-gen. Clinical Observation on Warm Acupuncture and Moxibustion Combined with Oblique-pulling Manipulation in the Treatment of Lumbar Disc Herniation[J]. Chinese Journal of Medicine, 2020, 26(12): 167-170.

[14] Lu Yu, Liu Zihao, Jing Tao, Fu Liyuan, Zhang Shu-shu. Diagnosis and treatment of perianal necrotizing fasciitis based on Yin and Yang syndrome differentiation in Traditional Chinese medicine surgery[J]. Journal of Tianjin University of Traditional Chinese Medicine, 2021, 40(04): 491-493.

[15] Feng Xiuli, Xu Qinghua, Zhao Xiaoyun, Liu Chang, Chen Yu, XU Wei. Treatment of Chronic Prostatitis by Electro - Acupuncture Combined with Warm - Needle Moxibustion: A Clinical Observation of 42 Cases. Journal of Shenyang Pharmaceutical University, 2013, 30(01): 35-39+62.

[16] Tang xiaoru bamboo leaf gypsum decoction for the treatment of 81 cases of fever[J]. Clinical Research of Traditional Chinese Medicine, 2020, 12(21): 129-130.

[17] Ma Le, XI Jianyuan, Bao Qiuyu. Clinical study of tori disinfection drink combined with Xiangpi Shengji ointment in the treatment of chronic skin ulcer[J]. China Medical Review, 2020, 17(25): 145-148.

[18] Liang Yongsheng, LI Chungeng, LI Wenhao, LIU Xuexian, Li Qin, Zheng Xue, Bo Hongmei, Guan Xin, Ma Jun, Xu Ying. Clinical study on the treatment of necrotizing fasciitis around anus with Todu Yiqi Yangyin Decoction[J]. Chinese Journal of Traditional Chinese Medicine and Emergency, 2021, 30(02): 249-252.

[19] Gu Feng. Clinical observation of Treating perianal necrotizing fasciitis with Decoction of Pyototoxin combined with operation $[\mathrm{J}]$. Chinese Journal of
Traditional Chinese Medicine and Emergency, 2017, 26(04): 668-670.

[20] Gu Feng. Clinical observation of Treating perianal necrotizing fasciitis with Decoction of Pyototoxin combined with operation[J]. Chinese Journal of Traditional Chinese Medicine and Emergency, 2017, 26(04): 668-670.

[21] Xie Jing, Gong Yixinyue, Ding Lisheng, Luo Pei, Qing Linsen. Research progress on clinical and pharmacological effects of Astragalus membranaceus and its active ingredients on sepsis[J]. Chinese Traditional and Herbal Medicines, 2021, 52(08): 2502-2510.

[22] Cao Rangfu. Effect of buzhong yiqi decoction on healing of anorectal diseases after operation[J]. Everyone Health (Academic Edition), 2014, 8(02): 55-56.

[23] Li L Z. Analysis of the effect of Shishida Bu Decoction on incision healing after anal carbuncle and anal fistula[J]. Chinese Medical Journal, 2014, 33(19): 131-132.

[24] Qin Yangyang. Clinical efficacy observation of wet healing in wound healing[C]. Nursing Society of Henan Province.2013 New progress of tumor nursing in Henan Province and the symposium of tumor specialized nurse training class. Nursing Association of Henan Province: Nursing Association of Henan Province, 2013: 88-89.

[25] Wei Zhenzhen, Fang Xiaoyan, Bai Ming, Miao Mingsan. Du Moxibustion Combined with Acupuncture in the Treatment of Acute Peripheral Facial Paralysis in One Case[J]. Journal of Hunan University of Traditional Chinese Medicine, 2019, 39(09): 1162-1165.

[26] Yu Xingyi, Li Yingju. Clinical application of wet healing method in chronic wound treatment $[\mathrm{J}]$. Journal of Kunming Medical University, 2019, 40(08): 146-148.

[27] Li Yu-bo, Chen Ju-hua, WANG Meng-di, DI K-qi, PANG Lim-ming. Effect of Kuilao Decoction on postoperative perianal necrotizing fasciitis[J]. Journal of Contemporary Medicine, 2020, 18(10): 211-212.

[28] Hong Lin, Pu LAN, Li Bingbing, Hu Zhengping, Ma Fengang, Cao Guoqiong. Advances in the study of chemical constituents, pharmacological effects and quality control of caryophylla broadleaf[J]. Guizhou Agricultural Sciences, 2019, 47(09): 122-125.

[29] Qiao Fengni, CAO Yanjian, Yang Xiangdong. Clinical efficacy of qingheat, detoxification, swelling and Pus decoction and kangfuxin liquid wet compress in promoting perianal necrotizing fasciitis wound healing[J]. Colorectal and Anal Surgery, 2021, 27(S1): 6-7.

[30] Hu Zhiliang, MAO Longfei, Li Chunwei. Treatment of perianal necrotizing fasciitis with operation combined with local sitting bath[J]. Chinese Medicine Research, 2020, 33(12): 21-24.

[31] Zhang X L, Yang G. Clinical study of Chinese herbal fumigation and washing after perianal necrotizing fasciitis[J]. Journal of Guiyang College of Traditional Chinese Medicine, 2013, 35(03): 175-177.

[32] Wang Lewei, Zhao Fengqin, Han Li, Wang Jianmin. Effect of alternating washing with hydrogen peroxide and compound Phellodendron solution on wound healing after perianal necrotizing fasciitis $[\mathrm{J}]$. Chinese Journal of Clinical Medicine, 2018, 30(06): 1154-1157. 
[33] Guo Ya-ping, MA Qian, HUANG Xiao-ming, Gao Ji-hua. Clinical study on wound recovery after anal fissure by "simmering pus and growing flesh" method[J]. Hebei Journal of Traditional Chinese Medicine, 2019, 34(02): 20-22.

[34] Xu Jiancheng, LI Fengsheng, Song Yihua, Wu Chunxiao, Wang $\mathrm{Na}$, Xue Li. Clinical study on promoting postoperative healing of necrotizing fasciitis with Ziyu Ointment "Simmering pus and growing flesh"[J]. Journal of Modern Medicine and Health Research, 2021, 5(10): 101-103.

[35] Nie Min, Lu Yao, Li Yu-bo, Lin Shusen, Li Chun-yu. Effect of meBO on wound after perianal necrotizing fasciitis[J]. Chinese Journal of Burn Innovation and Selection, 2021, 33(04): 296-298+302.

[36] Lian Hui-bin, Zhao Wei, Wang Ya-li, Xu Gang. Efficacy of mebo combined with mupiroxacin in the treatment of residual burn wounds[J]. Chinese Patent Medicine, 2016, 38(07): 1658-1660.

[37] Li Mengdi, Wang Ying. Study on chemical characteristics and mechanism of moxibustion[J]. Journal of Practical Traditional Chinese Medicine, 2017, 31(09): 87-90.

[38] Kan Yu, Zhang Xiao-ning, Yu Qing-quan, He Wei, Wang Xiao-yu, Wan Hong-ye, Su Yang-shuai, HU Ling, Zhang Zhi-yun, Chen Li-zhen, Jing Xiang-hong. Curative effect observation of acupuncture moxibustion combined with wound moxibustion postoperative healing of low anal fistula[J]. Acupuncture Research, 2019, 44(04): 288-292.

[39] Wang X Y. Clinical study on the intervention effect of moxibustion on wound healing after low anal fistula[D]. Hunan University of Chinese Medicine, 2015.

[40] Yang Zhen, Lin Fei, Zhang Qi. Clinical observation of acupuncture in treatment of postoperative constipation of mixed hemorrhoids in 65 cases[J]. Hunan Journal of Traditional Chinese Medicine, 2015, 31(12): 92-93.

[41] Gai Juanjuan, Zeng Science. Effect of Acupuncture at Dong Shi Qi point combined with Chinese medicine sit-bath on wound healing and related indexes after mixed hemorrhoids PPH $[\mathrm{J}]$. Journal of Modern Integrated Chinese and Western Medicine, 2020, 29(34): 3771-3775+3815.

[42] Zhang Dongping, Cao Jianchun, Ju up. Effect of Xuefengshengji Ointment on the expression of VEGF and PCNA in diabetic rats with skin ulcer[J]. Chinese Journal of Integrated Traditional and Western Medicine Surgery, 2018, 24(06): 737-742.

[43] Liu Xiao-tong, Shen Ruo-wu, Bian Ming-xin, Li Ling, Xia Yu-jun, Yang Yong-hua. Expression of VEGF, PDGF and bFGF in wound skin of rats[J]. Journal of Medical College of Qingdao University, 2016, 52(02): 209-211+214.

[44] Zhang Jin, Li Shi, Lan Fei, Guan Wei, Wang Bo. Effect of traditional Chinese medicine fumigation and acupuncture on postoperative wound healing in anorectal patients and its mechanism analysis[J]. Journal of Liaoning University of Traditional Chinese Medicine, 2019, 21(08): 107-110. 